\title{
Analyzing the Impact of Iridescence Strength on Animal Shape Recognition Using Computer Vision
}

\author{
Isabel Zhong ${ }^{1}$
}

${ }^{1}$ Santa Clara High School, Santa Clara, CA, USA

\section{ABSTRACT}

Iridescence, commonly found in nature, is the quality of a material to change colors depending on its interaction with light. For example, certain species of beetles distract predators with their iridescent exoskeletons that flash different colors depending on the angle the predator looks at them. Iridescent flowers, however, attract pollinators through their noticeable changes in color. Thus, it seems that iridescence can serve two contrasting purposes: concealment and attraction. The purpose of this project is to test whether different strengths in iridescence can impact its recognizability to animals and insects. Three different iridescent diffraction grating films were experimented with: $500 \mathrm{lines} / \mathrm{mm}, 532$ lines $/ \mathrm{mm}$, and 1000 lines $/ \mathrm{mm}$. Matte paper was used as a control. Four circular epoxy resin disks ( $8 \mathrm{~cm}$ diameter) and four square epoxy resin disks ( $7 \mathrm{~cm}$ side length) were created. Each diffraction grating film type and the matte paper was glued onto one disk of each shape. Photos of each disk were then taken at angles of 30, 45, 60, and 90 degrees in a photo booth and processed through a computer vision algorithm, the Ramer-Douglas-Peucker algorithm, to perform contour approximation. The results gathered cannot conclude that the strengths of iridescence have an impact on shape recognition and detectability due to limitations and potential errors. Nonetheless, the study can suggest that the specific usage of the Ramer-Douglas-Peucker algorithm for edge and area detection through OpenCV (an open-source computer vision library) is inadequate in imitating animal and insect visual systems when analyzing iridescence.

\section{Introduction}

Charles Darwin, the father of evolution, stated in his infamous book On the Origins of Species, "One general law, leading to the advancement of all organic beings, namely, multiply, vary, let the strongest live and the weakest die." (Darwin 1859). In other words, animals and plants possess biological features that will equip them with the best chance of survival throughout their life. While Darwin's theory has been proven to apply to the vast majority of biological systems, there is one biological feature that is still underexplored: iridescence.

Iridescence in nature, or the "interaction of light with biological tissues that are nanostructured to produce thin films or diffraction gratings," is found in flowers, insects, and many other plants and animals (Doucet \& Meadows 2009). Organisms that possess iridescence change colors depending on the angle and strength of the light it interacts with. However, the exact function of iridescence is inconsistent throughout nature. In certain cases, like iridescence in a male peacock's feather, scientists believe that it can help increase an organism's reproduction success by attracting those of the opposite sex. Likewise, scientists believe that iridescence in flower petals increases the flower's chances of survival by attracting pollinators. In other cases, such as in iridescent beetles, scientists predict that iridescence helps the beetles distract its predators. With that, researchers have begun to investigate the world of iridescence in hopes of finding a true answer to its ability to serve multiple functions.

Many researchers conducted their experiments through animal testing, but new technologies hold potential in replacing these techniques. In 1963, Lawrence Gilman Roberts introduced the idea of computer vision, or using computers to imitate human and animal visual systems (Roberts 1963). Over the past several decades, computer vision algorithms have become increasingly advanced, with abilities such as face recognition and object classification. These 
advancements spark the prospect of using computer vision as an alternative to animal testing for analyzing the features of iridescence.

\section{Literature Review}

\section{Functions of Iridescence in Nature}

Iridescence in plants and animals has been discussed by scientists for the past century, but advancements have been made the quickest in the recent decade due to technology. In 1909, Abbott Handerson Thayer, an American artist and naturalist, stated that "brilliantly changeable or metallic colors are among the strongest factors in animal's concealment" and that iridescence is made to "appear 'dissolved' in many depths and distances" (Thayer 1909). Thayer believed, from his observations as an artist, that nature balances the abilities of predators and prey where the predators kill off enough prey to stay alive as a race, but the prey develops protective features through natural selection and evolution to sustain their own population as well. Thayer claims that iridescence, or "brilliantly changeable or metallic colors" in a plant or animal, is a way for prey to confuse and hide from predators. In other words, he hypothesized that iridescence helps animals camouflage with their surroundings.

However, in 2009, a group of researchers published a paper in the Science journal focusing on the relationship between floral iridescence and its pollinators. The researchers hypothesized that floral iridescence, contrary to Thayer's belief, serves to attract pollinators (Whitney et al. 2009). After measuring the variation of color in an iridescent patch with spectroscopy for maximum and minimum iridescence, a bee hexagon color space (a model that represents the color perception of a bee) was used to calculate the spread of color loci. ${ }^{1}$ From these values, it was determined that the variation in color between iridescent objects was distinguishable by bees. Then, bees had to be tested to see whether or not they can differentiate between a matte and iridescent disk. After training the bees on iridescent disks with a sucrose reward, matte disks of the same color pigment were introduced, and the bees continued to visit the iridescent disks. Therefore, the research concludes that bees can distinguish between iridescent and non-iridescent objects on top of being able to recognize the color variation between iridescent objects. This research opens the possibility that flowers have adapted through evolution to either obtain or maintain their iridescent features to best attract pollinators, thus contradicting Thayer's idea that iridescence only serves to camouflage.

Building off the previously mentioned study, a group of researchers from the University of Bristol and the University of Cambridge wanted to analyze bees' perception of iridescence to a finer degree. They tested the differences between "perfect iridescence" created by an artificial grating film ${ }^{2}$, "imperfect" iridescence created by a flower petal, and "non-iridescence", or matte (Whitney et al. 2016). After creating different disks for each type of iridescence, the researchers trained the bees to locate a disk of a particular color. Then, each disk was laid out in the testing arena and the time it took for the bees to locate the same colored disk was recorded. According to their findings, the bees had the most trouble finding disks of perfect iridescence, then non-iridescence, and lastly imperfect iridescence. This research suggests that flowers would attract pollinators the best with imperfect iridescence, but the threshold in which "imperfect iridescence" lies remains unknown. The definition of "imperfect iridescence" is simply based on various flower petals, but is unable to be quantified. There must be a reason why not many species of flowers have iridescence, and the answer may lie in the specificity of "imperfection" that is required for optimal attraction to pollinators.

Similarly, a recent study published in 2018 took a more detailed spin on the previous studies. The researchers hypothesized that iridescence should impair shape recognition as its purpose in nature is to create "disruptive camouflage" described in Thayer's theory (Kjernsmo et al. 2018). Following a similar method to the research mentioned in

${ }^{1}$ color points received by UV, green, and blue photoreceptors

${ }^{2}$ Plastic film with small cuts to refract light 
the previous paragraph, these researchers created two disks for every type of iridescence (diffraction grating ${ }^{3}$, multilayer $^{4}$, floral iridescence ${ }^{5}$, and non-iridescence ${ }^{6}$ ), one of which was elliptical and the other was circular. They tested bees by using a sucrose reward and found that the "stronger" types of iridescence-diffraction grating and multilayer-deterred the bees significantly comparative to the non-iridescent disks whereas the more subtle floral iridescent disk did not impair the bees' shape recognition abilities. These findings suggest that iridescence could potentially serve multiple functions depending on the strength and type of iridescence. Stronger types may be able to deter predators whereas weaker types serve to be eye-catching and attract pollinators and mates. This study raises the question of whether or not different types of iridescence serve different functions in nature.

The studies mentioned above have been able to identify a significant gap: do all the different types or intensity of iridescence in nature serve the same purpose? And if not, what causes natural selection to arrive at the perfect intensity for flowers and animals? I hypothesize that there exist thresholds in nature where intensities of one threshold serve to attract and the intensities of another serve as disruptive camouflage. This led to the development of my leading question: how does the strength of iridescence in nature affect object and shape recognition amongst animals and insects?

\section{Computer Vision}

In many similar studies, the methods used were randomized testing on animals and insects. However, this was not a feasible method due to safety and ethical concerns. An alternative method was sought out: computer vision ${ }^{7}$. Lawrence Gilman Roberts, one of the founders of the Internet and the "father of computer vision", introduced the idea of using computers to identify three-dimensional objects by analyzing two-dimensional line drawings in his MIT Ph.D. thesis "Machine Perception of Three Dimensional Solids" in 1963 (Roberts 1963). In his thesis, he explored polygon recognition, computer transformations, and depth perception, thus initiating the field of computer vision for future researchers. However, his research only provided a basis for analyzing line drawings, not real-life images.

In 1982, David Marr, a neuroscientist at MIT, published Vision: A computational investigation into the human representation and processing of visual information. In this book, Marr identifies the necessity of following what he describes as the "computational theory," or the idea of a "bottom-up approach" where the fundamentals of computer vision must be laid down before the development of fancy algorithms will be useful (Marr 1982). He stresses the importance of "low-level" processing techniques such as edge detection, boundary detection, and grouping, urging other researchers to first focus on perfecting these techniques, which is central to the field of computer vision today. From then on, computer vision has grown to encompass numerous applications in robotics and medicine. For the purpose of computer vision in this experiment, only shape detection and classification are necessary. In the paper "Shape detection and classification using OpenCV and Arduino Uno" by Xhensila Poda and Olti Qirici, two professors at the University of Tirana, the researchers outline the procedures to conduct simple shape detection and classification (Poda \& Qirici). Using a development tool called OpenCV (Open Source Computer Vision), they list five steps for contour approximation: noise reduction ${ }^{8}$, finding the intensity gradient ${ }^{9}$, non-maximum suppression ${ }^{10}$, hysteresis

\footnotetext{
${ }^{3}$ Material that can split and separate light into beams that travel in various directions

${ }^{4}$ Material with multiple layers of reflectors

${ }^{5}$ Natural iridescence found in flower petals

${ }^{6}$ Matte

${ }^{7}$ Scientific field that explores the usage of computers to replicate human visual systems

${ }^{8}$ Using Gaussian filters to remove noise in images

${ }^{9}$ Analyzing gradients in pixels to identify the direction it is going in

${ }^{10}$ Checking the lines between pixels for local maxima, basically thinning out the edges
} 
thresholding ${ }^{11}$, and the contour detection algorithm ${ }^{12}$. The researchers conclude that this method is a practical and lowcost method for robotics technologies in the future. Thus, this method was a feasible and suitable procedure for the experiment in this study.

\section{Materials and Method}

Although the experiment in this paper is inspired by the vision of bumblebees and predatory animals in nature, it is not possible to test the hypothesis with actual live animals or insects due to the circumstances and amount of resources given. Instead, another approach can be taken where a computer vision algorithm will analyze high-resolution images of iridescent disks of varying diffraction gratings for changes in visible area in comparison to a matte disk, an alternative to observing an animal or insect's behavior and reaction to an object's visibility. Since varying diffraction gratings will diffract various amounts of light, it is hypothesized that the algorithm will have a harder time identifying the shape and amount of visible area for disks that diffract more light.

To narrow the scope of the experiment, only diffraction grating iridescence was experimented with. As mentioned before, diffraction grating film is a plastic film with slits. When light hits the film, the slits separate the light into multiple point sources with each slit being a point source. Each point source becomes the center of a light wave that is the shape of a circle, overlapping other waves of light from its neighboring point sources. As the waves constructively and destructively interfere, the film generates light of various amplitudes and results in the glittering effect (Jacobs 1996). A diffraction grating film of more lines/area is considered to be stronger, as it has more point sources and thus more overlapping points.

The study follows a simple ex post facto design as the characteristics of the iridescence are pre-existing. A true experimental research design is not practical given that iridescence in nature exists as is and is not an effect that evolves within plants or animals. An ex post facto design is a sufficient substitute by making observations of existing phenomenons. One disk of each shape (circle, square) and each strength of diffraction grating iridescence (500 lines $/ \mathrm{mm}, 532$ lines $/ \mathrm{mm}$, and 1000 lines $/ \mathrm{mm}$ ) was created and compared to the matte disk of the same shape through a computer vision algorithm.

\section{Safety Measures}

Safety protocols were closely followed throughout this experiment. As mentioned before, no live animals or insects were used in the experiment, contrary to the methods used in similar experiments conducted in the past, to ensure the safety of the conductor and any potential insects. Instead, a computer vision algorithm was used to imitate animal and insect vision. The disk creation process required the handling of epoxy and hardener. All safety requirements listed on the instructions provided by Let's Resin were adhered to. The experiment was conducted in a well-ventilated area with workspaces covered with plastic sheets. Disposable gloves and safety goggles were worn throughout the experiment and any spills were cleaned up immediately.

\section{Disk Creation}

For the experiment, films with diffraction gratings of 500 lines $/ \mathrm{mm}, 532$ lines $/ \mathrm{mm}$, and 1000 lines/mm were used. A matte disk with no diffraction grating film was used as the control disk. For the matte disk, the procedure was repeated but the film was substituted with printer paper. A circle of $8 \mathrm{~cm}$ in diameter and a square of $7 \mathrm{~cm}$ in side length was cut out of each type of film and paper to be glued on to epoxy disks later in the process.

${ }^{11}$ Differentiates "real-edges" and "non-edges" using minimum and maximum threshold levels

${ }^{12}$ Using the Ramer-Douglas-Peucker algorithm that reduces the number of points in a curve 
The disks were created following an established procedure (Kjernsmo et al. 2018). The epoxy resin was prepared in accordance with the provided instructions. In a bowl, $25 \mathrm{~mL}$ of Let's Resin crystal epoxy resin and $25 \mathrm{~mL}$ of Let's Resin crystal epoxy resin hardener were mixed for three minutes until clear and well combined. The mixture was left to sit for five minutes to allow air bubbles to dissipate. Then, one drop of Let's Resin liquid resin colorant in Sapphire Blue was added to make it easier to differentiate from the background during the photography process. The mixture was then poured into a silicone mold and left to set for 24 hours. This process was repeated eight times to create four circular disks ( $8 \mathrm{~cm}$ in diameter) and four square disks $(7 \mathrm{~cm}$ in side length).

Once the epoxy resin disks were set, they were removed from the silicone mold. Clear Matte Mod Podge was brushed onto each disk. Then, each cut out film/paper was placed onto its correspondingly shaped disk immediately and left to dry for 12 hours. At the end of the process, one disk of each of the following was created: matte square, 500 lines $/ \mathrm{mm}$ square, 532 lines $/ \mathrm{mm}$ square, 1000 lines $/ \mathrm{mm}$ square, matte circle, 500 lines $/ \mathrm{mm}$ circle, 532 lines $/ \mathrm{mm}$ circle, and 1000 lines $/ \mathrm{mm}$ circle.

\section{Photography}

After the disks were created, all square disks were placed in a mini photo studio tent with a white background and two LED studio lights set on opposite sides of the booth. A DSLR camera took high-resolution pictures of the disk from 30, 45, 60, and 90 degrees from the disk. The angles were set by adjusting a tripod in accordance with the reading returned on a digital protractor.

\section{Image Processing}

These photos were then processed through an OpenCV based Python algorithm that performed shape detection (Rosebrock 2019). Before any contour approximations, images were pre-processed to filter out noise and color. Each image is first pre-processed with a Gaussian blur ${ }^{13}$ with each Gaussian kernel set to a width and height of 7 pixels and a standard deviation of 1 . Then, the blurred image is converted from RGB to grayscale. The image is then processed through a popular multi-stage edge detection algorithm called Canny edge detection. Canny edge detection receives the input (the image) and two threshold values: threshold 1 and threshold 2. Threshold 1 represents the minimum intensity gradient ${ }^{14}$ and threshold 2 represents the maximum intensity gradient. Using a concept called hysteresis thresholding, the algorithm uses the two threshold values to determine whether or not an edge is a "non-edge" or a "classified-edge". "Non-edges" are discarded, leaving an outline of the inputted image. After edge detection, the edges of the image were dilated to $5 \times 5$ kernel $^{15}$, making the edges slightly thicker and closing off any gaps between fine edges.

For contour approximation, the algorithm used the Ramer-Douglas-Peucker algorithm, which is already implemented in OpenCV. The Ramer-Douglas-Peucker algorithm can take an epsilon value, or the maximum distance from the contour to the approximated contour, as an input. The epsilon value is a parameter for accuracy. The greater the value, the weaker the accuracy. However, too small of an epsilon level can result in image noise being detected. After testing various epsilon values, the optimal accuracy without sacrificing precision was found to be $2 \%$ of the arc length of each contour. With the epsilon value set as such, the algorithm is able to return an approximated contour area and number of points the contour has.

\footnotetext{
${ }^{13}$ Blurring an image using a Gaussian function to smooth out images and reduce image noise

${ }^{14}$ Directional change in intensity

${ }^{15}$ Small set of coordinates, also known as a structuring element
} 
Each image was processed through the algorithm and the area detected was recorded. All steps of the image processing are shown in Figure 1.

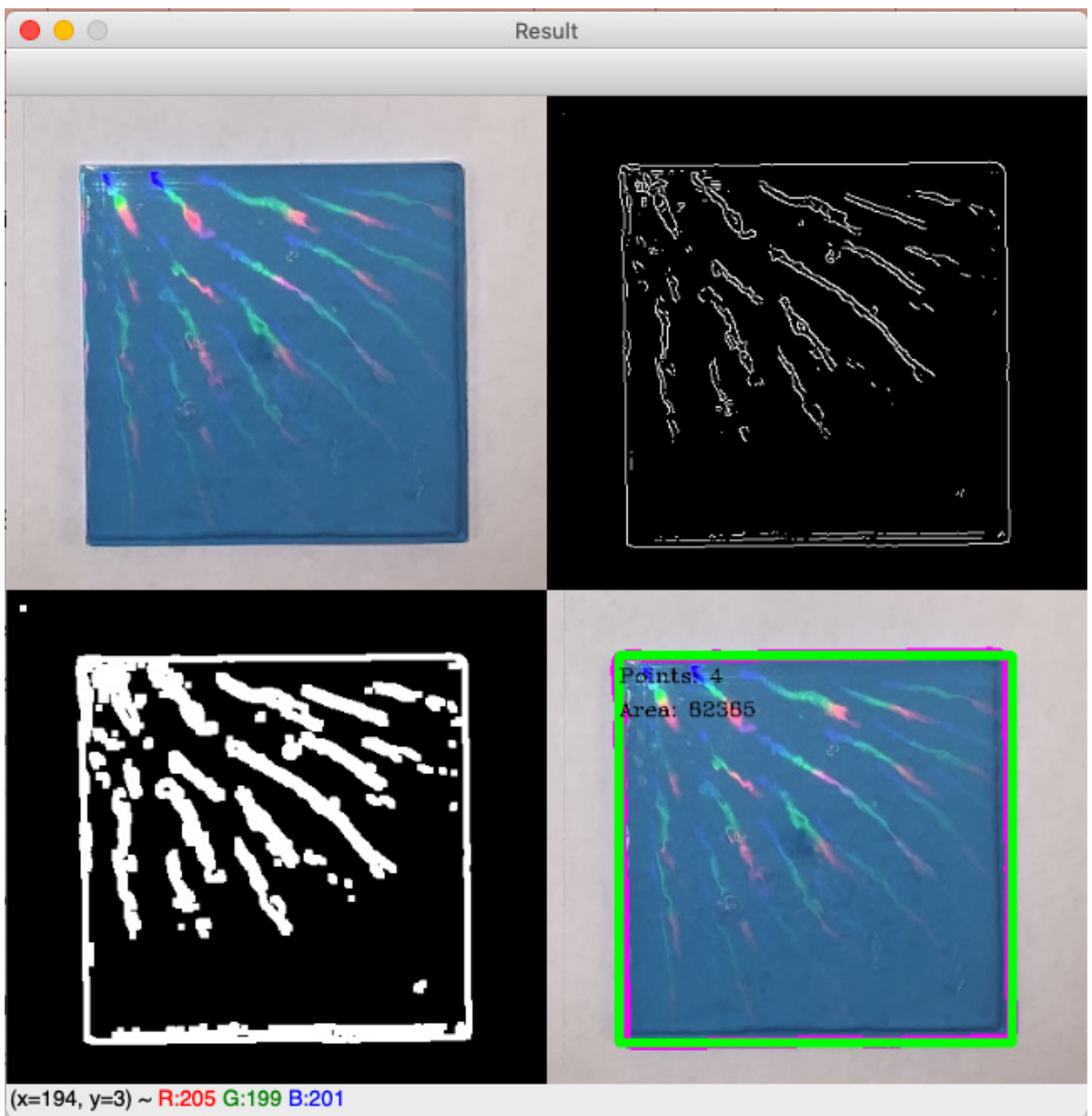

Figure 1. Image Processing Stages. Original image (top left), grayscale and Gaussian blurred image (top right), Canny edge detected image (bottom left), original image with results of the Ramer-Douglas-Peucker algorithm overlayed (bottom right).

\section{Results}

The data collected from the image processing stage through the computer vision algorithm is shown below in Figure 1. Each disk was processed through the algorithm, which returned the area of the detected shape in pixels. All disks were processed through the same algorithm with the setting of threshold 1 set to 50/255, setting of threshold 2 set to 50/255, and the minimum area detected set to 1000 pixels --the most ideal settings found to filter out noise in the background for these particular images. 
Table 1. Area Returned from Ramer-Douglas-Peucker algorithm

\begin{tabular}{|c|c|c|c|c|}
\hline Angle (degrees) & $\begin{array}{c}\text { Area of Square: } \\
\text { Matte (px) }\end{array}$ & $\begin{array}{c}\text { Area of Square: } 500 \\
\text { lines/mm (px) }\end{array}$ & $\begin{array}{c}\text { Area of Square: } 532 \\
\text { lines/mm (px) }\end{array}$ & $\begin{array}{c}\text { Area of Square: } 1000 \\
\text { lines/mm (px) }\end{array}$ \\
\hline 30 & 8460.50 & 8001.50 & 7923.75 & 9173.25 \\
\hline 45 & 10184.50 & 10748.25 & 10580.25 & 10922.50 \\
\hline 60 & 10311.50 & 9857.25 & 9981.00 & 10223.00 \\
\hline 90 & 11527.50 & 11138.75 & 11238.75 & 11131.00 \\
\hline Angle (degrees) & $\begin{array}{c}\text { Area of Circle: } \\
\text { Matte (px) }\end{array}$ & $\begin{array}{c}\text { Area of Circle: } 500 \\
\text { lines/mm (px) }\end{array}$ & $\begin{array}{c}\text { Area of Circle: } 532 \\
\text { lines/mm (px) }\end{array}$ & $\begin{array}{c}\text { Area of Circle: } 1000 \\
\text { lines/mm (px) }\end{array}$ \\
\hline 30 & 9745.00 & 9095.50 & 9692.25 & 7817.25 \\
\hline 45 & 11664.50 & 10526.25 & 10232.00 & 11289.50 \\
\hline 60 & 10564.25 & 10255.00 & 10375.25 & 9710.00 \\
\hline 90 & 11866.50 & 11463.50 & 11300.25 & 11475.50 \\
\hline
\end{tabular}

The adjustments of the tripod between each angle the photos were taken resulted in a difference in distance between the camera lens and the center of the disks. To account for this difference, the area returned of each iridescent disk picture was compared to the area of the matte disk picture taken at the same angle to create a percent error, or how much each iridescent disk's detected area differs from its corresponding angle's matte disk's detected area. This was calculated with the following equation for the values found in Table 1:

$$
\text { percent error }=\frac{\mid \text { area of matte disk }- \text { area of iridescent disk } \mid}{\text { area of matte disk }} \cdot 100
$$

Figure 2 shows the percent errors of each iridescent circle disks at each angle. Figure 3 below shows the percent errors of each iridescent square disk at each angle. 
Circle Disk Percent Error Analysis

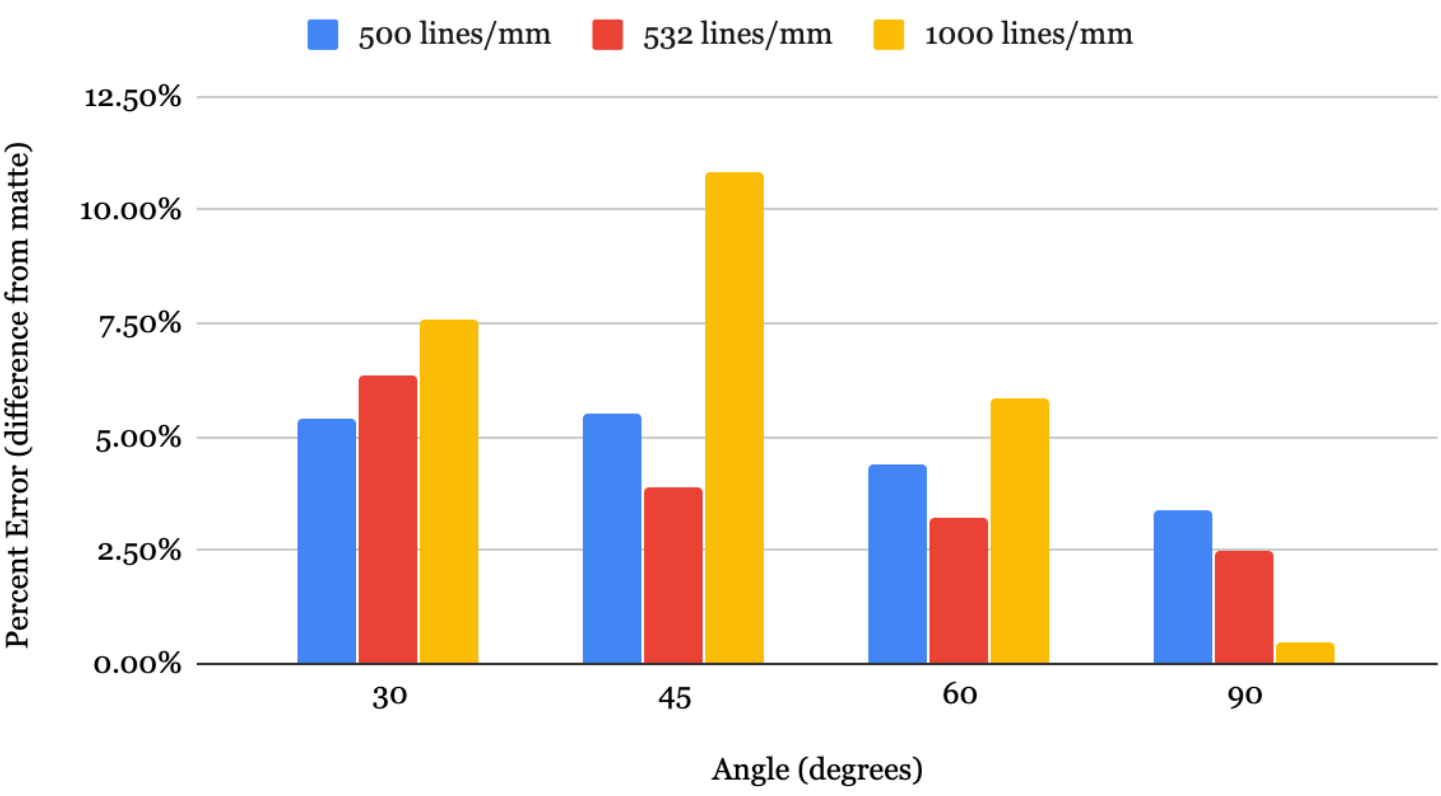

Figure 2. Graph of Percent Errors of Circle Disks

\section{Square Disk Percent Error Analysis}

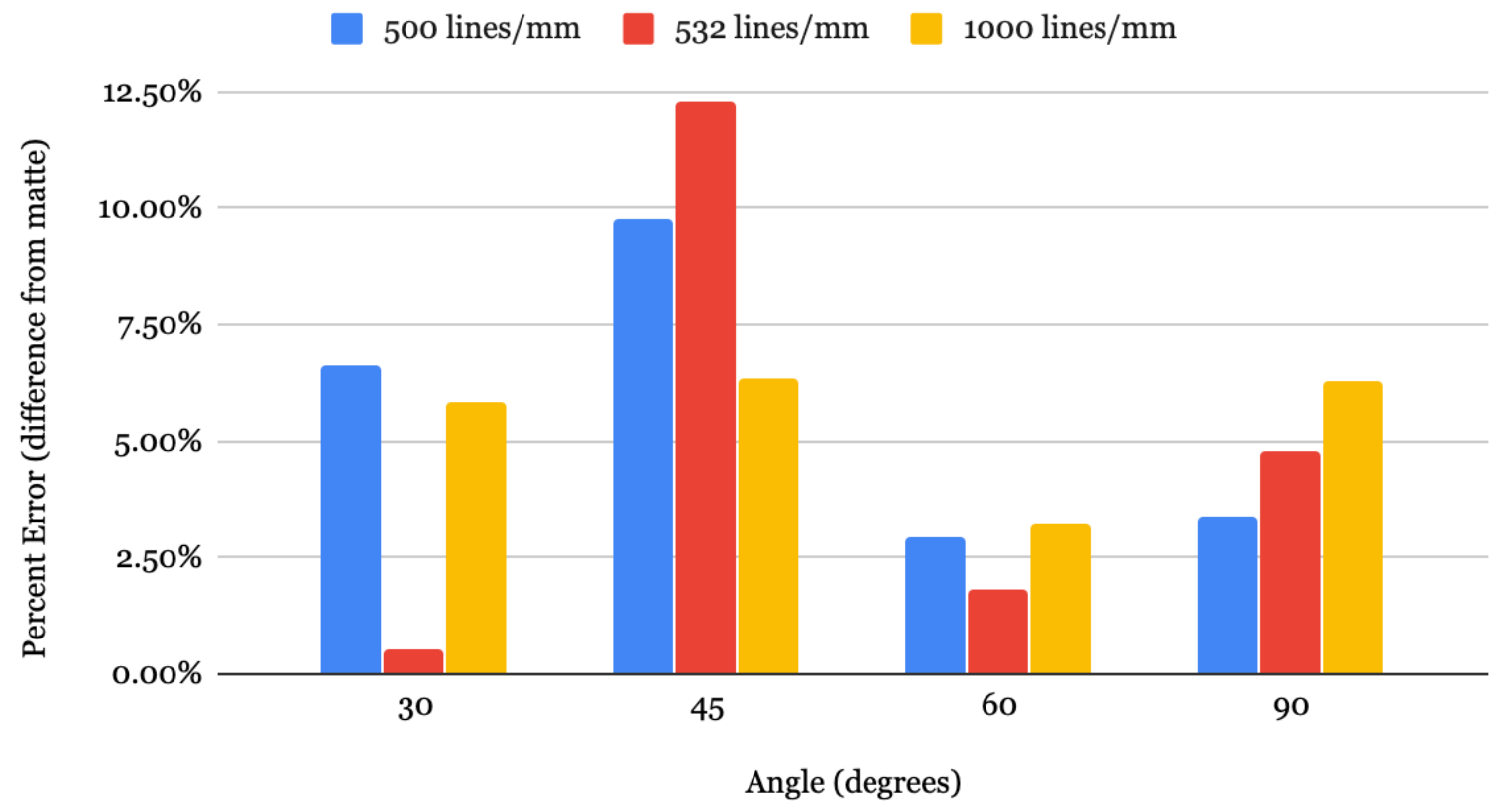

Figure 3. Graph of Percent Errors of Square Disks 


\section{Discussion}

The area of the iridescent disks detected by the algorithm differed from the matte disk in all cases. As illustrated in Figure 2 and Figure 3 above, the difference in detected area of each iridescent disk from the matte disk taken at the same angle and of the same shape has no observable pattern as iridescence strength increases.

For the circle disk samples, as shown in Figure 2, the percent errors increased as the strength in iridescence increased at 30 degrees. At 45 and 60 degrees, the strongest iridescence level (1000 lines $/ \mathrm{mm})$ had a greater difference than the weakest iridescence level (500 lines $/ \mathrm{mm}$ ), but the pattern was broken by the medium iridescence level (532 lines $/ \mathrm{mm}$ ) having a smaller difference than the weakest iridescence level. Lastly, at 90 degrees, the pattern was flipped from the pattern at 30 degrees, where the percent errors were decreasing as the strength in iridescence increased. The randomness of the results shows that there is no conclusive pattern between the strength of iridescence and recognized area in circle disks.

Similarly, for the square disk samples, there was no continuous pattern. As shown in Figure 3, at 30 degrees the ascending order of percent errors was the medium, highest, and lowest iridescence strength, respectively. At a 45 degree angle, the lowest strength iridescence still had a percent error greater than the highest strength iridescence, but the medium iridescence strength had a percent error greater than both the lowest and highest strength iridescence. At 60 degrees, the ascending order of percent errors was the medium, lowest, and highest iridescence strength, respectively. At 90 degrees, the ascending order of percent errors followed the ascending order of iridescence strength. Figure 2 shows that there is no significant pattern between an increase in iridescence strength and an increase in percent error, or difference from the matte disk.

The results found contradict the original hypothesis, that an increase in iridescence strength results in an increase in difference in the detected area from the matte disk. There were only two instances that supported the hypothesis, which was the circle disks at 30 degrees and the square disks at 90 degrees. However, since this pattern was not consistent for all angles and shapes, no conclusion or correlation can be drawn.

Multiple errors may have occurred during the experiment that may have affected the results. Firstly, due to a lack of resources, the variety of strengths in the iridescent films were limited to three different strengths: 500 lines $/ \mathrm{mm}$, 532 lines $/ \mathrm{mm}$, and 1000 lines $/ \mathrm{mm}$. Ideally, the experiment was intended to encompass a larger quantity of samples with a constant difference in strength between each consequential sample. However, diffraction grating films of those exact specifications are not commonly available commercially and were unfortunately out of the budget of the project. In previous similar studies, the method to analyze object or shape recognition of iridescent objects to animals was to use bumblebees (Bombus terrestris) with a sugar bait on randomized disks (Kjernsmo et al. 2018). This method was not chosen for this experiment because bees are difficult resources to get access to and there would be many safety risks to both the conductor of the experiment and the bees that are difficult to cater to.

Instead, another approach using computer vision was taken. However, there are multiple potential errors that arise with using computer vision. Computer vision algorithms analyze shape in an image based on the contours of an image after it has been converted into a binary image (black and white pixels only). This process is an oversimplified version of how animals and insects process shapes and colors, and could lead to discrepancies in the results. Bumblebee vision is based on two compound eyes and differs greatly from human vision (which computer vision algorithms are based on). They have a greater field of vision, a higher flicker threshold (clearer individual visual frames), different photoreceptors, and can see polarized light (Riddle 2016). These differences can contribute to inaccuracies in the data gathered and could potentially explain its inability to support the hypothesis of this experiment and its misalignment with the results of other studies.

In order to conclude that the iridescence strength has a relationship with shape recognition in bees, the experiment must be conducted with both a greater variety in iridescence strengths and using the established bee and sugar bait method previous studies have used, or an accurate alternative. 


\section{Conclusion and Future Directions}

The results of this study suggest that there is no evidence to support that there exists a relationship between iridescence strength and shape/object recognition amongst animals and insects. While the disk creation process went through smoothly, the results returned by the computer vision algorithm were not consistent enough to define a pattern or generate a strong claim. Nonetheless, the results yield contributions to a broader context. The results of this experiment suggest that computer vision methods are not ideal for imitating insect vision, particularly the usage of contour approximation and shape/area detection through OpenCV. Furthermore, the specific usage of the Ramer-DouglasPeucker algorithm for edge and area detection through OpenCV seems to be unable to distinguish between iridescent and non-iridescent objects. Other existing computer vision techniques may be better suitable for accomplishing the goal of this project.

Thus, future studies should abstain from using computer vision to imitate insect vision during the analysis of shapes and objects unless new technologies or approaches develop substantial algorithms and techniques that can accurately replicate vision from compound eyes. Using bumblebees to test for differences in shape/object recognition was the initial goal of this project, as many previous similar studies have done. Due to safety and resource limits, it was not possible.

Furthermore, future experiments should explore greater varieties in iridescence strengths, preferably with a greater range and a constant difference between strengths. A wider range of strengths would offer a greater possibility of finding differences between weaker and stronger iridescence levels. A constant increase between the strengths would allow for a more accurate analysis of the existence of patterns between iridescence strength and object recognition. From a larger scope, a test of different types of iridescence could also lead to a greater understanding of animal and insect vision. In this experiment, only diffraction grating films were used, but other forms of iridescence like multilayer iridescence or natural iridescence of flowers or insects could provide fascinating data.

Another interesting perspective to take would be to repeat the experiment without the usage of blue dye in the experiment. Since the disks were dyed blue and placed on a white background during the photography process, the edge detection of the disk itself was based on the contrasting colors between the blue disk and the white background. This brings up a question of whether or not a change in background colors would impact the computer vision algorithm's edge detection abilities. Experimenting with different backgrounds such as naturistic backgrounds or backgrounds of the same color as the disk may yield important discoveries in regards to the shape detection of iridescent objects through a dimensional and reflective lighting perspective rather than an edge and area perspective pursued in this paper.

Although the results of this study were unfortunately unable to prove a relationship between iridescence strengths and shape recognition in animals and insects, the study suggests that there is still a possibility of a relationship if the experiment is conducted again after minimizing the limitations and taking a different approach from OpenCV computer vision. Such an experiment can further the body of knowledge in regard to animal and insect vision, potentially leading to significant advances and applications in robotics and biological fields.

\section{Acknowledgements}

I would like to thank Alumilite Corporation and Let's Resin for kindly donating epoxy and resin supplies and Rainbow Symphony for donating diffraction grating films. 


\section{References}

Contour Features. (n.d.). Open CV. Retrieved from https://docs.opencv.org/trunk/dd/d49/tutorial py contour fea$\underline{\text { tures.html }}$

Darwin, C. (1859, November 24). On The Origin of Species. Retrieved from http://www.talkorigins.org/faqs/origin.html

Doucet, S. M., \& Meadows, M. G. (2009, February 23). Iridescence: a functional perspective. Retrieved from https://royalsocietypublishing.org/doi/10.1098/rsif.2008.0395.focus\#d3e178

Jacobs, S. F. (1996). How A Diffraction Grating Works. Wyant College of Optical Sciences. Retrived from wp.optics.arizona.edu/sfjacobs/wp-content/uploads/sites/47/2016/06/DiffractionGratings.pdf

Kjernsmo, K., Hall, J. R., Doyle, C., Khuzayim, N., Cuthill, I. C., Scott-Samuel, N. E., \& Whitney, H. M. (2018, May 25). Iridescence impairs object recognition in bumblebees. Retrieved from https://www.nature.com/articles/s41598-018-26571-6\#Bib1

Marr, D. (1982). A Computational Investigation into the Human Representation and Processing of Visual Information. W.H. Freeman and Company.

Poda, X., \& Qirici, O. (n.d.). Shape detection and classification using OpenCV and Arduino Uno. Retrieved from http://ceur-ws.org/Vol-2280/paper-19.pdf

Riddle, S. (2016, May 20). How Bees See And Why It Matters. Retrieved from http://www.beeculture.com/beessee-matters

Roberts, L. G. (1963, January 1). Machine perception of three-dimensional solids. Retrieved from https://dspace.mit.edu/handle/1721.1/11589

Rosebrock, A. (2016, February 8). OpenCV Shape Detection. PyImageSearch. Retrieved from www.pyimagesearch.com/2016/02/08/opencv-shape-detection/

Thayer, G. H., Theodore Roosevelt Hunting Library \& Thayer, A. H. (1909) Concealing-coloration in the animal kingdom; an exposition of the laws of disguise through color and pattern: being a summary of Abbott H. Thayer's discoveries. New York, The Macmillan Co. Retrieved from the Library of Congress, https://lccn.loc.gov/10000866

Whitney, H., Kolle, M., Andrew, P., Chittka, L., Steiner, U., \& Glover, B. (2009). Floral Iridescence, Produced by Diffractive Optics, Acts as a Cue for Animal Pollinators. Science, 323(5910), new series, 130-133. Retrieved from http://www.jstor.org/stable/20177140

Whitney, H. M., Reed, A., Rands, S. A., Chittka, L., \& Glover, B. J. (2016, March 21). Flower Iridescence Increases Object Detection in the Insect Visual System without Compromising Object Identity. Retrieved from https:/www.ncbi.nlm.nih.gov/pmc/articles/PMC4819513/ 